\title{
Measurements of Magneto-Rayleigh-Taylor Instability Growth during the Implosion of Initially Solid Al Tubes Driven by the 20-MA, 100-ns Z Facility
}

\author{
D. B. Sinars, ${ }^{1}$ S. A. Slutz, ${ }^{1}$ M. C. Herrmann, ${ }^{1}$ R. D. McBride,${ }^{1}$ M. E. Cuneo, ${ }^{1}$ K. J. Peterson, ${ }^{1}$ R. A. Vesey, ${ }^{1}$ C. Nakhleh, ${ }^{1}$ \\ B. E. Blue, ${ }^{2}$ K. Killebrew, ${ }^{2}$ D. Schroen, ${ }^{2}$ K. Tomlinson, ${ }^{1}$ A. D. Edens, ${ }^{1}$ M. R. Lopez, ${ }^{1}$ I. C. Smith, ${ }^{1}$ J. Shores, ${ }^{1}$ V. Bigman, ${ }^{1}$ \\ G. R. Bennett, ${ }^{1}$ B. W. Atherton, ${ }^{1}$ M. Savage, ${ }^{1}$ W. A. Stygar, ${ }^{1}$ G. T. Leifeste, ${ }^{1}$ and J. L. Porter ${ }^{1}$ \\ ${ }^{1}$ Sandia National Laboratories, P.O. Box 5800, Albuquerque, New Mexico 87185, USA \\ ${ }^{2}$ General Atomics, San Diego, California 92121, USA \\ (Received 4 August 2010; published 25 October 2010)
}

\begin{abstract}
The first controlled experiments measuring the growth of the magneto-Rayleigh-Taylor instability in fast $(\sim 100 \mathrm{~ns})$ Z-pinch plasmas are reported. Sinusoidal perturbations on the surface of an initially solid Al tube (liner) with wavelengths of $25-400 \mu \mathrm{m}$ were used to seed the instability. Radiographs with $15 \mu \mathrm{m}$ resolution captured the evolution of the outer liner surface. Comparisons with numerical radiation magnetohydrodynamic simulations show remarkably good agreement down to $50 \mu \mathrm{m}$ wavelengths.
\end{abstract}

DOI: 10.1103/PhysRevLett.105.185001

PACS numbers: 52.58.Lq, 52.30.Cv, 52.35.Py, 52.65.Kj

This Letter describes the first controlled measurements of the growth of the magneto-Rayleigh-Taylor (MRT) instability in fast $(\sim 100 \mathrm{~ns}) \mathrm{Z}$ pinches. The MRT instability [1-4] is ubiquitous in pinch plasmas in which the $\vec{j} \times \vec{B}$ force is used to compress matter. In these experiments a Zpinch plasma was formed from an initially solid metal tube commonly referred to as a liner. In cylindrical liner implosions the MRT instability arises at the outer plasmavacuum interface, where the driving magnetic pressure plays a role analogous to a light fluid pushing on a heavy fluid (the plasma liner) as in the classical fluid RayleighTaylor instability. The MRT instability is considerably more complex in part because the driving current is not confined to the surface but diffuses into the liner, allowing resistive heating of the liner and distributing the magnetic pressure. Applications of fast Z-pinch implosions include fusion schemes in which a cylindrical liner containing fusion fuel (deuterium and/or tritium) is directly compressed by the magnetic pressure to the conditions needed to produce yield $[5,6]$. The major factor limiting the integrity of the liner used to compress the fusion fuel is breakup due to the MRT instability.

There are many published experiments studying the growth of the Rayleigh-Taylor instability in laser- or radiation-driven high energy-density plasmas going back to the 1990s (e.g., Refs. [7,8]) and continuing to this day. Very few controlled MRT instability growth experiments exist in the literature. The only sub- $\mu$ s data we found are from wire-array tests using wires with axial modulations in the initial mass per unit length [9]. Solid liner implosions evolve differently than wire-array implosions, which are dominated by the ablation of order half of the initial mass into the array interior before the implosion begins. The remaining published controlled studies were done on multi- $\mu$ s generators in which the imploding liners have significant material strength and remain in liquid or solid states for much of the implosion [10]. By contrast, in fast $(\sim 100 \mathrm{~ns})$ solid liner implosions strong shocks can develop and the liner is in the plasma state for much of the implosion. Because of the lack of high-quality data for the sub- $\mu$ s regime, the magnetohydrodynamic physics packages of simulation codes (e.g., LASNEX [11], HYDRA [12], GORGON [13]) are not as well validated as the radiation-hydrodynamics packages, despite the fact that they have been used for years to model instability growth in sub- $\mu$ s Z-pinch implosions (e.g., Refs. [13-15]).

In this Letter we present data from a series of controlled MRT growth experiments on the 20 MA, 100 ns Z facility in which radiography was used to quantify the growth of the instability. The hardware configuration and current drive for these experiments are summarized in Fig. 1.
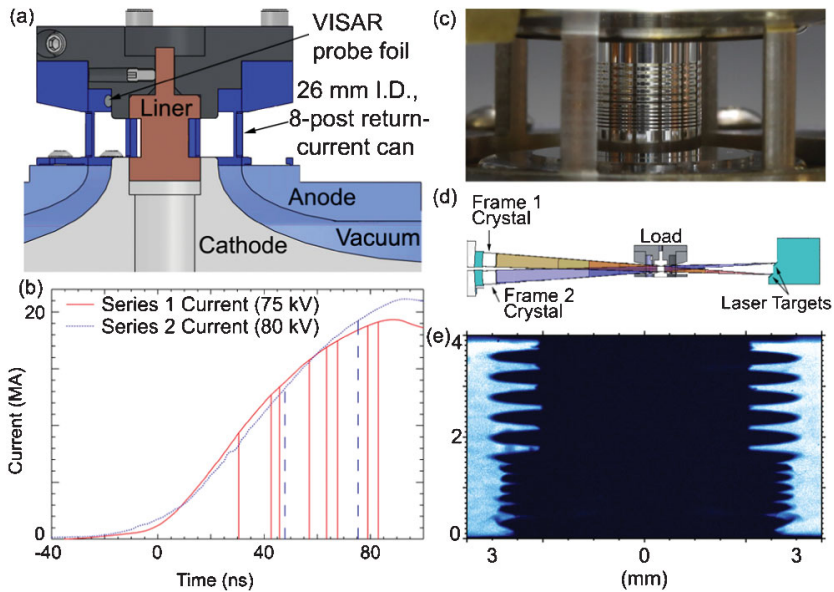

FIG. 1 (color). Description of the experiments. (a) Halfsection diagram of the power-feed hardware surrounding the load. (b) Measured currents for the experiments with radiograph times overlaid as vertical bars. (c) Photo of a target inside the return-current can. (d) Schematic of the 2-frame backlighter illustrating the $3^{\circ}$ angle above or below horizontal. (e) Example radiograph data ( $t=83 \mathrm{~ns}$ from series 1$)$. 
Cylindrical liners made of Al 1100 alloy were placed inside of an 8-post, $26 \mathrm{~mm}$ inner diameter return-current structure (can). The liners had an outer radius of $3.168 \mathrm{~mm}$, a wall thickness of $292 \mu \mathrm{m}$, and contained a $2 \mathrm{~mm}$ diameter tungsten rod inside on the liner axis. The large returncurrent can radius was chosen to reduce the azimuthal variations in the static magnetic field caused by the 8 posts to $<1 \%$. The rod was used to quench radiation produced by the plasma stagnation on axis. The liner dimensions are similar to those being considered for future inertial confinement fusion experiments [6]. The MRT instability was seeded by machining sinusoidal perturbations with peakto-valley amplitudes that were 5\% of the wavelength. In the first series of experiments, the liners used wavelengths with $\lambda=200$ and $400 \mu \mathrm{m}(10,20 \mu \mathrm{m}$ amplitudes, respectively). The instability growth was recorded at 8 different times during the implosion using two-frame, monochromatic $6.151 \mathrm{keV}$ backlighting [16]. The timing of the radiographs with respect to the current is shown in Fig. 1(b). An example radiograph image is shown in Fig. 1(e). A second series of experiments used targets with wavelengths of $25,50,100$, and $200 \mu \mathrm{m}$ and a large flat region in the backlighter field of view. A one-frame $6.151 \mathrm{keV}$ diagnostic [17] was used to provide a horizontal $\left(0^{\circ}\right)$ view to prevent shadowing of the bubbles by the spikes in the small-wavelength perturbations.

We chose 200- and 400- $\mu \mathrm{m}$ wavelengths for the first series to match LASNEX simulations of liners with a nominal $60 \mathrm{~nm}$ surface roughness amplitude, which show wavelengths of 200-400 $\mu \mathrm{m}$ when the liner mass nears the axis [6]. These wavelengths are also well resolved by the $6.151 \mathrm{keV}$ backlighter, which has a measured spatial resolution of $15 \mu \mathrm{m}$ (the 10\%-90\% width of an edge-spread measurement, which corresponds to the full width at half maximum of a Gaussian point-spread function). Finally, the simulations predicted observable differences between the 200 and $400 \mu \mathrm{m}$ waves such as jet formation.

Portions of a sequence of radiographs obtained during the first series are shown in Fig. 2. We show only one side of the liner, but all of the images were highly correlated azimuthally as shown in Fig. 1(e), and the features showed only small variations from one wave to another. Early in time the current diffuses into the liner and heats up its outermost layer, which ablates and forms an expanding plasma. The expansion is largely normal to the liner surface, so that the ablated plasma is focused by the sinusoidal curvature. In the higher-curvature $200 \mu \mathrm{m}$ waves the focusing results in an early reversal of the apparent position of the peaks and valleys that eventually culminates in the distinct, narrow jets seen in the later radiographs. Eventually the material in the jets diffuses to a low enough areal density to be invisible to the $6.151 \mathrm{keV} x$ rays and/or is compressed back onto the liner by the increasing magnetic pressure. The plasma temperature in the jets (e.g., in frame 5 of 8 ) is estimated in simulations to be about $30 \mathrm{eV}$, (a) $200 \mu \mathrm{m}$ Data

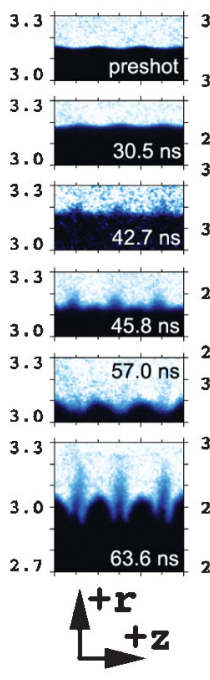

FIG. 2 (color). Expanded views of portions of the radiographs from series 1 [timing in Fig. 1(b)]. (a) Central three of the five $\lambda=200 \mu \mathrm{m}$ waves. (b) Two of the six $\lambda=400 \mu \mathrm{m}$ waves. The $\pm 3^{\circ}$ backlighter viewing angle limits the visible axial extent of the bubbles, which are completely shadowed by the spikes in the last two $\lambda=200 \mu \mathrm{m}$ frames.

and in the valleys about $100 \mathrm{eV}$. By contrast, in the $400 \mu \mathrm{m}$ data the amplitude decreases to $17 \mu \mathrm{m}$ in frame 1 due to ablation, but thereafter the amplitude of the perturbation grows continuously without prominent jet formation. As we will demonstrate, the ablation, jet formation, and amplitude growth were successfully predicted by our LASNEX simulations.

A plot of the amplitude versus time for the $400 \mu \mathrm{m}$ data is shown in Fig. 3. We estimate an error in the cross timing between the radiograph times and the measured current at $\pm 1.0 \mathrm{~ns}$. The error in the amplitude is dominated by the shot-to-shot uncertainty in the magnification of about $\pm 3 \%$ and the statistical variation in the amplitude from one feature to another, for a total uncertainty of about $\pm 5 \%$. Also plotted is the amplitude of simulated radiographs of 4-wave LASNEX calculations. The simulations used a uniform density with an initial surface roughness that approximated the surface roughness of the $\mathrm{Al}$, which was measured to have roughly $30 \mathrm{~nm}$ root mean square roughness. The surface roughness is not random, but has azimuthal striations with a periodicity of $1.25 \mu \mathrm{m}$ from the diamond tooling used to machine the targets (the simulations used an $\sim 8 \mu \mathrm{m}$ period). The simulations capture the overall trend and late-time amplitudes remarkably well, though they underpredict the early-time amplitude growth.

We briefly compare these results to simple theory. The equations describing the MRT instability in a cylindrical geometry can be linearized to yield an analytic solution [1-4]. The resulting amplitude $\eta$ grows exponentially as $\eta=\eta_{0} \exp G(t)$, where $G(t)=\int_{0}^{t} \gamma\left(t^{\prime}\right) d t^{\prime}$ and 

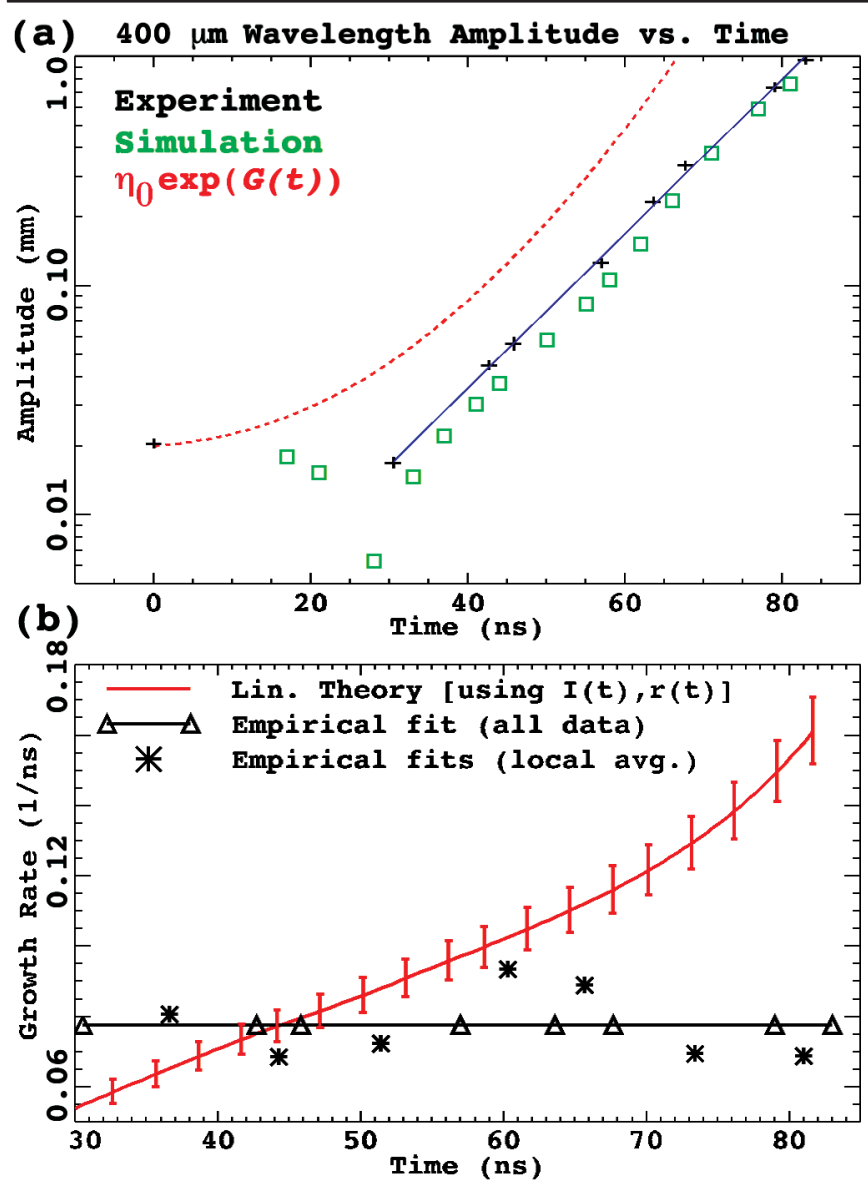

FIG. 3 (color). Analysis of $\lambda=400 \mu \mathrm{m}$ data from series 1 . (a) Peak-to-valley amplitude vs time for experiments, simulations, and linear theory. The extent of the crosses represents the measurement error. The line represents an exponential fit to the data. (b) The instantaneous growth rate $[\gamma(t)]$ is shown in red. An exponential growth rate averaged over all the data is shown in black, along with asterisks showing the local growth rate calculated for each pair of points in the data.

$\gamma^{2}=k g(k=2 \pi / \lambda)$. Calculating the acceleration $g$ from the magnetic pressure gives

$$
[\gamma(t)]^{2}=k \frac{\mu_{0}}{8 \pi^{2}} \frac{[I(t)]^{2}}{[R(t)]^{2}} \frac{1}{\rho(\Delta r)},
$$

where $I$ is the measured current, $R$ is the measured minimum radius, $\rho$ is the density, and $\Delta r$ is the initial liner thickness. The average experimental growth rate over all of the data in the 30-82 ns range is coincidentally well fit by an exponential with a constant growth rate. Figure 3(a) shows that the simple analytic theory overpredicts the amplitude and does not capture the reduction in amplitude due to ablation. The instantaneous growth rates shown in Fig. 3(b) are similar near 40-45 ns, but the analytic growth rate thereafter exceeds the empirical rate. [The error in $\gamma(t)$ plotted in Fig. 3 includes our $\pm 3 \%$ error on current [18] and the $\pm 3 \%$ error in $R$.] For long wavelengths, the linearization of the equations is an increasingly poor approximation once the amplitude reaches $\sim 1 / k$ $(\sim 60 \mu \mathrm{m}$ for $\lambda=400 \mu \mathrm{m})$ [3]. From Fig. 3(a) we see the amplitude reaches $60 \mu \mathrm{m}$ at about $t=47 \mathrm{~ns}$. After this time, higher-order terms in the equations become important and the growth rate calculated numerically is slower than predicted by the linearized equations. Our detailed numerical calculations using LASNEX capture this slower amplitude growth and lower growth rate.

A second series studied the development of the MRT instability in both flat regions (perturbed only by surface roughness) and regions with 25-, 50-, 100-, and 200- $\mu \mathrm{m}$ seeded perturbations. Radiographs were obtained at $t=$ 47.8 and 75.4 ns. The earlier radiograph, shown in Fig. 4, captured the ablation and jetting of low-density ablated plasma seen in the $\lambda=200 \mu \mathrm{m}$ data from the first series. LASNEX simulations [Fig. 4(b)] again were able to match the jet structures and evolution of the $\lambda=200 \mu \mathrm{m}$ region. The agreement with the $\lambda=100 \mu \mathrm{m}$ data is also quite good except for slight shape differences in the jets. The simulations begin to deviate from the experimental data in the $\lambda=50 \mu \mathrm{m}$ region, and the $\lambda=25 \mu \mathrm{m}$ region shows relatively poor agreement with the simulations in that the spike structures extend farther out radially and are more easily distinguishable in the simulations than the experiment. The disagreement may be due in part to not fully resolving the roughness of the liner and in part because LASNEX is a 2D code and the data appear to be increasingly three dimensional at smaller scales.

The experimental and simulated radiographs were Abel-inverted line by line using the cold opacity of $\mathrm{Al}$ at $6.151 \mathrm{keV}\left(102.6 \mathrm{~cm}^{2} / \mathrm{g}\right)$ to infer the radial density profile (Fig. 4). The inversion highlights subtle differences in the

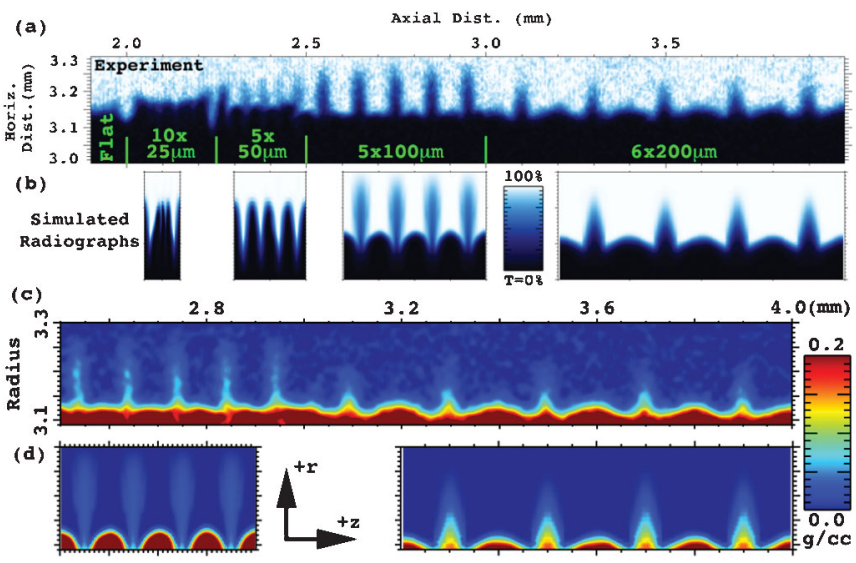

FIG. 4 (color). Analysis of the $t=47.8 \mathrm{~ns}$ radiograph from series 2 [Fig. 1(b)]. (a) A portion of the experimental radiograph that captures the ablation and jetting from 25-, 50-, 100-, and $200-\mu \mathrm{m}$ wavelength regions. (b) Simulated radiographs from four different LASNEX simulations of each region. (c) Experimental density contour plot obtained from an Abel inversion of the radiography data. (d) Simulated density contour plot obtained from an Abel inversion of the simulated radiographs. Densities $>0.2 \mathrm{~g} / \mathrm{cm}^{3}$ were set to 0.2 in (c) and (d). 


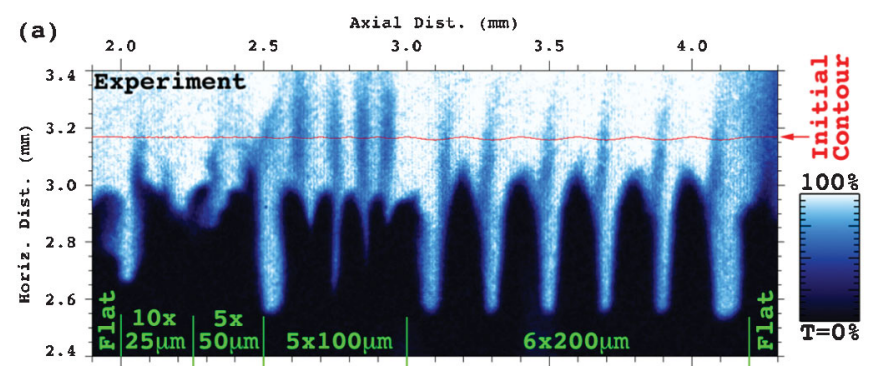

(b)
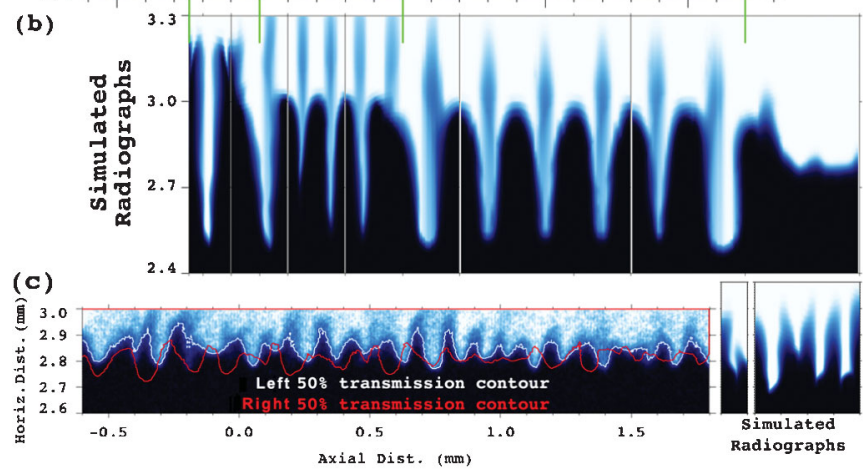

FIG. 5 (color). Analysis of the $t=75.4 \mathrm{~ns}$ radiograph from series 2 [Fig. 1(b)]. (a) Experimental radiograph of the perturbed region with the initial contour overlaid. (b) A mosaic of simulated radiographs from 6 different LASNEX calculations. (c) Experimental and simulated radiographs of the flat (unperturbed) region. The $50 \%$ transmission contour from the other side of the target is overlaid in red.

$\lambda=100 \mu \mathrm{m}$ region, most notably that the jets are lower density and slightly wider in the simulations than in the experiments. Integrating the amount of mass at radii $>3.14 \mathrm{~mm}$ (the base of the jet in the $100 \mu \mathrm{m}$ region), we estimated $2.8 \mu \mathrm{g} /$ jet experimentally and $2.0 \mu \mathrm{g} / \mathrm{jet}$ in the simulations. The same mass estimates in the $200 \mu \mathrm{m}$ region were 2.3 and $2.0 \mu \mathrm{g} / \mathrm{jet}$, respectively. The initial liner mass/length is $1500 \mu \mathrm{g}$ per $100 \mu \mathrm{m}$ height, so $2.8 \mu \mathrm{g}$ represents about $0.18 \%$ of the total mass over the $100 \mu \mathrm{m}$ wavelength height (about $0.5 \mu \mathrm{m}$ thickness). This agreement is remarkable given that prior to the experiments there was uncertainty as to whether the jets predicted by LASNEX would even exist. Indeed, we have not yet successfully reproduced these jets in other codes.

Experimental and simulated radiographs for $t=75.4 \mathrm{~ns}$ are shown in Fig. 5. Again the agreement between the experiment and simulations is remarkably good down to $\lambda=50 \mu \mathrm{m}$. The picture in Fig. 5(b) is a mosaic of six different calculations and illustrates a challenge we have in modeling the MRT instability. To capture small-scale details requires fine zoning ( $>20$ axial zones per wave), but such calculations over any significant axial extent require significant computing resources. We need to assess whether modeling small scales is necessary to capture details when the liner is close to the axis, or if we can get by with larger scales. The reason is that smaller wavelengths tend to fold over and merge with each other to form larger-scale structures $[14,15]$, as is just beginning to happen in the $\lambda=100 \mu \mathrm{m}$ region of Fig. 5 .

Data from the initially unperturbed (flat) region of the liner are shown in Fig. 5(c). The overlaid contour from the other side of the radiograph demonstrates that the data are not two dimensional. By contrast, the perturbed regions remain symmetric at this time. Accurately simulating this will require three-dimensional codes.

We note that beryllium has a lower opacity at $6.151 \mathrm{keV}$ $\left(2.24 \mathrm{~cm}^{2} / \mathrm{g}\right)$, which may allow future in-flight liner areal density measurements even at small radii. We are continuing to use this data as a benchmarking tool for other codes (e.g., HYDRA and GORGON). The success of the LASNEX modeling is encouraging in that we previously used the same methodology to design a magnetized liner inertial fusion target for the $\mathrm{Z}$ facility that may be capable of yields of $\gtrsim 100 \mathrm{~kJ}$ [6]. These results increase the credibility of those calculations, which suggest that liners with outer radius to thickness ratios of 4-6 maintain sufficient integrity to compress the fusion fuel.

We thank the Z, Z-Beamlet, diagnostics, target, and hardware teams for their support of this work, which was partially funded by Laboratory Directed Research and Development funds at Sandia. Sandia is a multiprogram laboratory operated by Sandia Corporation, a Lockheed Martin Company, for the National Nuclear Security Administration under DE-AC04-94AL85000.

[1] E. G. Harris, Phys. Fluids 5, 1057 (1962).

[2] E. Ott, Phys. Rev. Lett. 29, 1429 (1972).

[3] D. D. Ryutov, M. S. Derzon, and M. K. Matzen, Rev. Mod. Phys. 72, 167 (2000).

[4] A. R. Miles, Phys. Plasmas 16, 032702 (2009).

[5] T. J. Nash et al., Phys. Plasmas 12, 052705 (2005).

[6] S. A. Slutz et al., Phys. Plasmas 17, 056303 (2010).

[7] B. A. Remington et al., Phys. Rev. Lett. 67, 3259 (1991).

[8] B. A. Remington et al., Phys. Rev. Lett. 73, 545 (1994).

[9] B. Jones et al., Phys. Rev. Lett. 95, 225001 (2005).

[10] R. E. Reinovsky et al., IEEE Trans. Plasma Sci. 30, 1764 (2002).

[11] G. B. Zimmerman and W.L. Kruer, Comments Plasma Phys. Controlled Fusion 2, 51 (1975).

[12] M. M. Marinak et al., Phys. Plasmas 8, 2275 (2001).

[13] J. P. Chittenden et al., Plasma Phys. Controlled Fusion 46, B457 (2004).

[14] M. R. Douglas, C. Deeney, and N.F. Roderick, Phys. Plasmas 5, 4183 (1998).

[15] D. L. Peterson et al., Phys. Plasmas 3, 368 (1996).

[16] G. R. Bennett et al., Rev. Sci. Instrum. 79, 10E914 (2008).

[17] D. B. Sinars et al., Rev. Sci. Instrum. 75, 3672 (2004).

[18] T. C. Wagoner et al., Phys. Rev. ST Accel. Beams 11, 100401 (2008). 